\title{
Pilocarpus spp.: A survey of its chemical constituents and biological activities
}

\author{
Ana Paula Santos, Paulo Roberto Hrihorowitsch Moreno* \\ Laboratório de Química de Produtos Naturais, Departamento de Química Orgânica, Instituto de Química, \\ Universidade de São Paulo
}

*Correspondência:

P. R. H. Moreno

Laboratório de Química de Produtos

Naturais

Instituto de Química, USP

Av. Prof. Lineu Prestes, 748. Bloco 11 T

Cidade Universitária

05508-000 São Paulo - SP

E-mail: prmoreno@iq.usp.br
Pilocarpus species have been exploited as the only source of the imidazole alkaloid pilocarpine (used in glaucoma treatment), since its isolation up to the present. Almost all Pilocarpus species are native from Brazil. Because of the medicinal importance of pilocarpine, several of them are in the path of extinction. Other secondary metabolites, such as coumarins, flavonoids and terpenes, were described for Pilocarpus species. In this review the secondary metabolites, other than pilocarpine, isolated from Pilocarpus species and their biological activities were compiled. Although the variety of structures and the importance of the biological activities described in literature for Pilocarpus species this is an unexploited field of research in Natural Products and Pharmacology.

\section{INTRODUCTION}

Pilocarpus Vahl is one of the 150 genus that compose the Rutaceae family and it was first known in Europe from the reports of the early scientific expeditions made to Brazil (Gabriel Soares de Souza, in 1570, Guilherme Piso, in 1637, and Georg Marcgrave, in 1638).
Several Pilocarpus species are popularly known by the name of "jaborandi" which was derived from the tupiGuarani language, ya-mbor-endi (the one who causes mouth-dripping) (Holmsted et al., 1975). Several Rutaceae species are important due to their economical value as food, ornamental and medicinal uses. Some of the most common examples are listed in Table I.

TABLE I - Examples of the several uses of Rutaceae species

\begin{tabular}{lll}
\hline Species & Usage & References \\
\hline Citrus spp. & Horticulture, volatile oils & Albuquerque, 1968 \\
Pilocarpus spp. & Medicinal (pilocarpine) & Albuquerque, 1968 \\
Zanthoxylum spp. & Carpentry & Corrêa, 1975 \\
Euxilophora paraensis Huber & Carpentry & Loureiro, Silva, 1968 \\
Esenbeckia febrifuga St. Hil. & Medicinal (febrifuge) & Albuquerque, 1968 \\
& & Corrêa, 1969 \\
\hline
\end{tabular}


The Pilocarpus genus was defined in 1797 with the descriptions of the species $P$. racemosus, however the position of the new taxon was not indicated (Skorupa, 1996). The initial conflicts in the systematic studies of the Rutaceae family imposed difficulties to classify this genus (Pirani, 1999; Albuquerque, 1985).

The most complete study about this genus was performed by Kaastra (1982) that kept the classification of Pilocarpus accordingly to Engler and recognized the genus Raulinoa Cowan as part of the subtribe Pilocarpineae (Scheme 1).

The Pilocarpus species are distributed in a large region of the American continent from Southern CentralAmerica (Mexico) until the Southern part of the SouthAmerican continent (Argentina, Paraguay). There are 16 species described for this genus accordingly to Skorupa (1996). From these 13 can be found in Brazil, and 11 occur exclusively in the Brazilian territory (Table II).

These plants started to be studied concerning their pharmacological properties only in 1873 , when it was confirmed that "jaborandi" was a potent stimulant of the secretory system, showing sialagogue, diuretic and diaphoretic activities (Corrêa, 1969). Since then, infusions of "jaborandi" leaves have been used for the treatment of fevers, stomatitis, bronchitis, gout, psoriasis, kidney diseases and several others illnesses (Holmsted et al., 1975).

After the isolation of the imidazole alkaloid pilocarpine (Figure 1, compound 1), in 1875 (Gerrard, 1875; Hardy, 1875), and its employment in ophthalmology (Weber, 1876), the "jaborandi" reached the status of one of the most important plants in the Brazilian flora (Pinheiro, 1997).

Besides alkaloids, a number of secondary metabolites have been reported in Pilocarpus species such as coumarins (Amaro-Luis et al., 1990), flavonoids (Bertrand et, al 2001), hydrocarbons (Skorupa et al., 1998; Negri et al., 1998), triterpenes (Andrade Neto et al., 1994) and volatile oils (Craveiro et al., 1979).

The main goal of this review is to point out the importance of the Pilocarpus spp. as a source of secondary metabolites in Rutaceae with a potential pharmacological activity.

\section{SECONDARY METABOLITES OF PILOCARPUS SPP.}

\section{Alkaloids}

The "jaborandi" alkaloids are mainly of the imidazole group and it is thought that $L$-histidine is the biosynthetic precursor of the imidazole moiety (Battersby, Openshaw, 1953; Brochmann-Hanssen et al., 1975; Cordell, 1981; Dewick, 2000).

Pilocarpine and other related alkaloids were isolated from several Pilocarpus species (Table III). Although the structural resemblance, only pilocarpine posses CNS activity, as a direct cholinergic which stimulates the parasympathetic system (bladder, tear ducts, sudoriferous and salivary glands). This alkaloid is the elected drug in the glaucoma treatment (Korolkovas, 1996) and the sialagogue property of pilocarpine has been exploited to treat the xerostomy (dry mouth) caused by the radio- or chemotherapy of throat cancer or those in the head area (Miller, 1993; Valdez et al., 1993).

Others alkaloids derived from $L$-tryptophan were also isolated from Pilocarpus species. Some examples are $N, N$-dimethyl-5-methoxy-triptamine (10) and $N, N$ dimethyl-triptamine (11) (known as DMT) obtained from

$\begin{array}{lllll}\text { Family } & \text { Subfamily } & \text { Tribe } & \text { Subtribe } & \text { Genus } \\ \text { Rutaceae } & \text { Rutoideae } & \text { Galipeae } & \text { Pilocarpineae } & \text { Pilocarpus } \\ & & \text { (Cuspariea) } & & \text { Essenbeckia } \\ & \text { Dictyolomatoideae } & \text { Zanthoxyleae } & \text { Metrodorea } \\ & \text { Spathelioideae } & \text { Ruteae } & \text { Raulinoa } \\ \text { Toddalioideae } & \text { Boroneae } & \text { Galipea * } \\ & \text { Flindersioideae } & \text { Diosmeae } & \text { Raputia * } \\ \text { Citroideae } & & \text { Neoraputia * } \\ & & & \end{array}$

SCHEME 1 - Systematic classification of Pilocarpus genus in Rutaceae. 
TABLE II - Distribution of Pilocarpus species (Skorupa, 1996)

\begin{tabular}{|c|c|}
\hline Species & Localization \\
\hline Pilocarpus alatus C. J. Joseph ex Skorupa & Brazil (Pará and Mato Grosso) \\
\hline Pilocarpus carajaensis Skorupa & Brazil (Pará) \\
\hline Pilocarpus demerarae Sandwith & Guiana Inglesa \\
\hline Pilocarpus giganteus Engler & $\begin{array}{l}\text { Brazil (Bahia, Espírito Santo, Minas Gerais, Rio de Janeiro and São } \\
\text { Paulo) }\end{array}$ \\
\hline Pilocarpus grandiflorus Engler & Brazil (Alagoas, Bahia and Espírito Santo) \\
\hline Pilocarpus jaborandi Holmes & Brazil (Ceará and Pernambuco) \\
\hline Pilocarpus manuensis Skorupa & Peru (Manu National Park) \\
\hline Pilocarpus microphyllus Stapf ex Wardleworth & Brazil (Maranhão, Mato Grosso, Pará and Piauí), Surinam \\
\hline Pilocarpus pauciflorus St. Hilaire & $\begin{array}{l}\text { Brazil (Paraná, Santa Catarina, Rio de Janeiro and São Paulo) } \\
\text { (subSpecies pauciflorus) } \\
\text { Brazil (Bahia) (subspecies clavatus) } \\
\text { Brazil (subSpecies organensis) }\end{array}$ \\
\hline Pilocarpus pennatifolius Lemmaire & $\begin{array}{l}\text { Brazil (Bahia, Goiás, Mato Grosso and Mato Grosso do Sul, Minas } \\
\text { Gerais, Paraná, Pernambuco, Rio Grande do Sul, Santa Catarina } \\
\text { and São Paulo), Paraguay (Caazapá, Paraguarí), Argentina } \\
\text { (Missiones) }\end{array}$ \\
\hline Pilocarpus peruvianus (Macbride) Kaastra & Bolivia, Brazil (Acre, Rodônia e Mato Grosso) and Peru \\
\hline Pilocarpus racemosus Vahl & $\begin{array}{l}\text { Mexico, Antilles, Colombia, Venezuela and British Guyana } \\
\text { (subspecies racemosus) } \\
\text { Venezuela and Colombia (subspecies goudotianus) } \\
\text { Costa Rica and El Salvador (subspecies viridulus) }\end{array}$ \\
\hline Pilocarpus riedelianus Engler & Brazil (Alagoas, Bahia, Espírito Santo, Paraíba and Pernambuco) \\
\hline Pilocarpus spicatus St. Hilaire & $\begin{array}{l}\text { Brazil (Bahia, Espírito Santo, Rio de Janeiro and São Paulo) } \\
\text { (subspecies spicatus) } \\
\text { Brazil (Bahia) (subspecies longeracemosus) } \\
\text { Brazil (Ceará, Paraíba and Pernambuco) (subspecies aracatensis) }\end{array}$ \\
\hline Pilocarpus sulcatus Skorupa & Brazil (Bahia and Minas Gerais) \\
\hline Pilocarpus trachyllophus Holmes & Brazil (Bahia, Ceará, Minas Gerais and Piauí) \\
\hline
\end{tabular}

P. organensis leaves; both have a CNS activity causing hallucinations (Balsam, Voigtländer, 1978).

Dictamine (14) a quinoline alkaloid obtained from $P$. grandiflorus posses a structural relationship with coumarins and, probably because of this resemblance, this substance exhibited a photosensitizing activity (Ashwood et al., 1982).

\section{Coumarins}

Coumarins are secondary metabolites biosynthesized from $p$-coumaric acid (a phenylalanine derived). Besides the simple coumarins, like scopolentin (28), in Pilocarpus species are also found pyrano- or furanocoumarins either with linear - xantyletin (13), chalepin (32) and bergapten (20) — or with an angular structure - allo-xantoxilletol (17) and 3-methoxyangelicin (24). Some coumarins found in Pilocarpus species may also be $O$-prenylated - donatin (18), imperatorin (23) - or $C$-prenylated — osthole (30).

Although studies on the isolation of secondary products from Pilocarpus sp. have been performed for a long time, the isolation of coumarins has only been reported just over a decade ago from the leaves of P. goudotianus (Amaro-Luis et al., 1990). The efficacy of the jaborandi infusions in the treatment of skin diseases such as psoriasis and vitiligo might be related with this class of natural products. Nowadays, several coumarins (particularly the 8-methoxy-psoralen (21)) have been used for skin repigmentation (Manderfeld et al., 1997).

Some coumarins also showed good results as germination inhibitors (MACÍAS et al., 1993), insecticide 
TABLE III - Alkaloids derived from Pilocarpus species and some related biological activities

\begin{tabular}{|c|c|c|c|}
\hline Alkaloid & Species & Activities & References \\
\hline pilocarpine (1) & $\begin{array}{l}\text { P. jaborandi } \\
\text { P. microphyllus } \\
\text { P. pennatifolius } \\
\text { P. racemosus } \\
\text { P. trachyllophus } \\
\text { P. riedelianus }\end{array}$ & $\begin{array}{l}\text { Sialagogue } \\
\text { Diuretic } \\
\text { Febrifuge }\end{array}$ & $\begin{array}{l}\text { Gerrard, } 1875 \\
\text { Hardy, } 1875 \\
\text { Jowett, } 1900 \\
\text { Mester, } 1973 \\
\text { Payo Hill et al., } 1995 \\
\text { Andrade Neto et al., } 1996 \\
\text { Guerreiro et al., } 2003\end{array}$ \\
\hline isopilocarpine (2) & $\begin{array}{l}\text { P. jaborandi } \\
\text { P. microphyllus } \\
\text { P. pennatifolius }\end{array}$ & - & $\begin{array}{l}\text { Gerrard, } 1875 \\
\text { Hardy, } 1875 \\
\text { Mester, } 1973\end{array}$ \\
\hline pilocarpidine (3) & P. jaborandi & - & Jowett, 1900 \\
\hline isopilocarpidine (4) & P. jaborandi & - & Jowett, 1900 \\
\hline pilosine (5) & $\begin{array}{l}\text { P. jaborandi } \\
\text { P. microphyllus }\end{array}$ & - & $\begin{array}{l}\text { Pyman, } 1912 \\
\text { Voigtländer, Rosenberg, } 1959 \\
\text { Tedeschi et al., } 1973\end{array}$ \\
\hline isopilosine (6) & P. jaborandi & - & Voigtländer, Rosenberg, 1959 \\
\hline epiisopilosine (7) & P. jaborandi & - & Tedeschi et al., 1973 \\
\hline epiisopiloturine (8) & P. jaborandi & - & Tedeschi et al., 1973 \\
\hline 13-nor-7(11)-dehidro-pilocarpine (9) & P. trachyllophus & - & Andrade Neto et al., 1996 \\
\hline$N, N$-dimethyl-5-methoxy-triptamine (10) & P. organensis & Hallucinogen & $\begin{array}{l}\text { Balsam, Voigtländer, } 1978 \\
\text { Riba et al., } 2002\end{array}$ \\
\hline$N, N$-dimethyl-triptamine (11) & P. organensis & Hallucinogen & $\begin{array}{l}\text { Balsam, Voigtländer, } 1978 \\
\text { Riba et al., } 2002\end{array}$ \\
\hline plastydesmine (12) & P. grandiflorus & - & De Souza et al., 2003 \\
\hline (1H)-4-methoxy-2-quinolone (13) & P. grandiflorus & - & De Souza et al., 2003 \\
\hline dictamine (14) & P. grandiflorus & $\begin{array}{l}\text { Antifungic } \\
\text { Vasorelaxing } \\
\text { Photosensitizing } \\
\text { Anticoagulant }\end{array}$ & $\begin{array}{l}\text { Asswood-Smith et al., } 1982 \\
\text { Yu et al., } 1992 \\
\text { De Souza et al., } 2002 \\
\text { Su et al., } 1998\end{array}$ \\
\hline
\end{tabular}

(Calcagno et al., 2002; Ngwedson et al., 2003; Manderfeld et al., 1997), antioxidant (Shaw et al., 2003; Hoult, Paya, 1996), and against Chagas' disease (Pavão et al., 2002; Mafezoli et al., 2000). Synthetic coumarins derived from seselin (35) exhibit potential activity against HIV (Huang et al., 1994).

The known coumarins isolated from Pilocarpus species and their respective structures are listed in Table IV and Figure 2.

\section{Flavonoids}

Flavonoids represent one of the most important groups of phenolic natural products and, like coumarins, they are also biosynthesized from $L$-phenylalanine. These metabolites are obtained from Citrus species (Rutaceae) from seeds, fruits, flowers and leaves (Middleton, 1984). Among others, the suggested activities of flavonoids are: analgesic (Thirugnanasambanthan et al., 1990), sedative (Medina et al., 1997; Marder \& Paladini, 2002), antiinflammatory (Pathak et al., 1991).

The antioxidant activity of these substances is due to the ability of reducing free radical formation and to scavenge free radicals. Accordingly to Makersby (1997), the neurodegenerative Alzheimer's disease manifestation is related to the production of these species in the organism. In this sense, flavonoids can be considered as promising drugs in the treatment of this disease (Kim et al., 2002).

Hesperidin (46) was isolated from P. trachyllophus (Bertrand et al., 2001). This substance is currently isolated from other Rutaceae, such as Citrus and Zanthoxylum 
<smiles>CCC1C(=O)OCC1Cc1cncn1C</smiles>

(1)<smiles>CC[C@@H]1C(=O)OCC1Cc1cncn1C</smiles>

(2)<smiles>CCC1C(=O)OC[C@@H]1Cc1cnc[nH]1</smiles>

(3)<smiles>CC[C@@H]1C(=O)OC[C@@H]1Cc1cnc[nH]1</smiles>

(4)<smiles>Cn1cncc1C[C@@H]1COC(=O)[C@H]1C(O)c1ccccc1</smiles>

(5)<smiles>Cn1cncc1C[C@@H]1COC(=O)[C@H]1[C@H](O)c1ccccc1</smiles>

(6)<smiles>Cn1cncc1C[C@@H]1COC(=O)[C@H]1[C@H](O)c1ccccc1</smiles>

(7)<smiles>COc1ccc2[nH]cc(CCN(C)C)c2c1</smiles>

(10)<smiles>Cn1cnc(C[C@@H]2COC(=O)[C@H]2[C@H](O)c2ccccc2)c1</smiles>

(8)<smiles>CN(C)CCc1c[nH]c2ccccc12</smiles>

(11)<smiles>CC1=C(Cc2cncn2C)COC1=O</smiles>

(9)

(12)<smiles>COC1CC(=O)Nc2ccccc21</smiles>

(13)<smiles>COc1c2ccccc2nc2occc12</smiles>

(14)

FIGURE 1 - Chemical structures of the alkaloids isolated from Pilocarpus species.

species (Kanes et al., 1993; Horowitz, Gentili, 1963; Arthur et al., 1956). This flavonoid has various pharmacological activities and some of them are antiinflammatory, bactericidal, fungicidal, antiviral, abortive, analgesic and antipyretic (Garg et al., 2001). Recently, hesperidin was obtained from Valeriana species ("the Valium of $19^{\text {th }}$ century") and also exhibited sedative and sleep-enhancer properties (Marder et al., 2003).
The following flavonoids, listed in Table V, were isolated from $P$. trachyllophus leaves.

\section{Terpenes}

Several reports dealt with the terpenes present in Pilocarpus species, mostly in volatile oils. Terpenes are biosynthesized from IPP (isopentenyl diphosphate), 
TABLE IV - Coumarins isolated from Pilocarpus species and some related biological activities

\begin{tabular}{|c|c|c|c|}
\hline Coumarin & Species & Activity & References \\
\hline xantyletin (15) & $\begin{array}{l}P \text {. goudotianus } \\
P \text {. racemosus } \\
P . \text { riedelianus }\end{array}$ & $\begin{array}{l}\text { Antifungic } \\
\text { Anti-PAF } \\
\text { Allelochemical } \\
\text { Insecticide }\end{array}$ & $\begin{array}{l}\text { Amaro-Luis et al., } 1990 \\
\text { Teng et al., } 1992 \\
\text { Macías et al., } 1993 \\
\text { Calcagno et al., } 2002\end{array}$ \\
\hline xanthoxyletin (16) & P. goudotianus & $\begin{array}{l}\text { Anti-PAF } \\
\text { Allelochemical } \\
\text { Insecticide }\end{array}$ & $\begin{array}{l}\text { Amaro-Luis et al., } 1990 \\
\text { Teng et al., } 1992 \\
\text { Macías et al., } 1993 \\
\text { Calcagno et al., } 2002\end{array}$ \\
\hline luvangetin (17) & $\begin{array}{l}\text { P. goudotianus } \\
\text { P. racemosus }\end{array}$ & $\begin{array}{l}\text { Allelochemical } \\
\text { Insecticide }\end{array}$ & $\begin{array}{l}\text { Amaro-Luis et al., } 1990 \\
\text { Compagnone, Rodriguez, } 1993 \\
\text { Macías et al., } 1993 \\
\text { Calcagno et al., } 2002\end{array}$ \\
\hline donatin (18) & P. goudotianus & $\begin{array}{l}\text { Allelochemical } \\
\text { Insecticide }\end{array}$ & $\begin{array}{l}\text { Amaro-Luis et al., } 1990 \\
\text { Macías et al., } 1993 \\
\text { Calcagno et al., } 2002\end{array}$ \\
\hline allo-xanthoxilletol (19) & P. goudotianus & $\begin{array}{l}\text { Allelochemical } \\
\text { Insecticide }\end{array}$ & $\begin{array}{l}\text { Amaro-Luis et al., } 1990 \\
\text { Macías et al., } 1993 \\
\text { Calcagno et al., } 2002\end{array}$ \\
\hline bergapten (20) & $\begin{array}{l}\text { P. goudotianus } \\
\text { P. racemosus }\end{array}$ & $\begin{array}{l}\text { Photosensitizing } \\
\text { Bactericide } \\
\text { Allelochemical } \\
\text { Insecticide }\end{array}$ & $\begin{array}{l}\text { Amaro-Luis et al., } 1990 \\
\text { Compagnone, Rodriguez, } 1993 \\
\text { Macías et al., } 1993 \\
\text { Manderfeld } \text { et al., } 1997 \\
\text { Calcagno et al., } 2002\end{array}$ \\
\hline 8-methoxy-psoralen (21) & $\begin{array}{l}\text { P. goudotianus } \\
\text { P. racemosus }\end{array}$ & $\begin{array}{l}\text { Photosensitizing } \\
\text { Vasorelaxing } \\
\text { Bactericide } \\
\text { Allelochemical } \\
\text { Insecticide }\end{array}$ & $\begin{array}{l}\text { Amaro-Luis et al., } 1990 \\
\text { Compagnone, Rodriguez, } 1993 \\
\text { Macías et al., } 1993 \\
\text { Manderfeld et al., } 1997 \\
\text { Chiou et al., 2001 } \\
\text { Calcagno et al., } 2002\end{array}$ \\
\hline isopimpinellin (22) & P. goudotianus & $\begin{array}{l}\text { Vasorelaxing } \\
\text { Bactericide } \\
\text { Allelochemical } \\
\text { Insecticide }\end{array}$ & $\begin{array}{l}\text { Amaro-Luis et al., } 1990 \\
\text { Macías et al., } 1993 \\
\text { Chiou et al., } 2001 \\
\text { Calcagno et al., } 2002 \\
\text { Ngwendson et al., } 2003\end{array}$ \\
\hline imperatorin (23) & P. goudotianus & $\begin{array}{l}\text { Vasorelaxing } \\
\text { Allelochemical } \\
\text { Insecticide }\end{array}$ & $\begin{array}{l}\text { Amaro-Luis et al., } 1990 \\
\text { Su et al., } 1998 \\
\text { Chiou et al., } 2001 \\
\text { Ngwendson et al., } 2003\end{array}$ \\
\hline tricocline (24) & P. goudotianus & - & Amaro-Luis et al., 1990 \\
\hline$(+)$-elisin (25) & $\begin{array}{l}\text { P. goudotianus } \\
\text { P. grandiflorus }\end{array}$ & - & $\begin{array}{l}\text { Amaro-Luis et al., } 1990 \\
\text { De Souza et al., } 2003\end{array}$ \\
\hline $\begin{array}{l}\text { 3,6-dimethoxy-angelicin (26) } \\
\text { 3-methoxy-angelicin (27) } \\
\text { scopoletin (28) }\end{array}$ & $\begin{array}{l}P \text {. riedelianus } \\
P \text {. riedelianus } \\
P \text {. riedelianus } \\
P \text {. grandiflorus }\end{array}$ & $\begin{array}{l}- \\
\text { Antioxidant }\end{array}$ & $\begin{array}{l}\text { Müller et al., } 1993 \\
\text { Müller et al., } 1993 \\
\text { Müller et al., } 1993 \\
\text { De Souza et al., } 2002 \\
\text { Shaw et al., } 2003\end{array}$ \\
\hline $\begin{array}{l}\text { pimpinellin (29) } \\
\text { osthole (30) }\end{array}$ & $\begin{array}{l}\text { P. riedelianus } \\
\text { P. riedelianus }\end{array}$ & $\begin{array}{l}\text { - } \\
\text { Anticoagulant } \\
\text { Vasorelaxing }\end{array}$ & $\begin{array}{l}\text { Müller et al., } 1993 \\
\text { Müller et al., } 1993 \\
\text { Chiou et al., } 2001\end{array}$ \\
\hline $\begin{array}{l}\text { meranzin hidrate (31) } \\
\text { sphondin (32) }\end{array}$ & $\begin{array}{l}\text { P. riedelianus } \\
\text { P. riedelianus }\end{array}$ & $\begin{array}{l}- \\
-\end{array}$ & $\begin{array}{l}\text { Müller et al., } 1993 \\
\text { Müller et al., } 1993\end{array}$ \\
\hline
\end{tabular}


TABLE IV - Coumarins isolated from Pilocarpus species and some related biological activities (continuation)

\begin{tabular}{|c|c|c|c|}
\hline Coumarin & Species & Activity & References \\
\hline auraptene (33) & P. riedelianus & Antitumoral & $\begin{array}{l}\text { Müller et al., } 1993 \\
\text { Murakami et al., } 1997\end{array}$ \\
\hline chalepin (34) & P. spicatus & $\begin{array}{l}\text { Anti-Chagas' } \\
\text { disease }\end{array}$ & Pavão et al., 2002 \\
\hline seselin (35) & P. riedelianus & Anti-HIV & $\begin{array}{l}\text { Guerreiro et al., } 2001 \\
\text { Huang et al., } 1994\end{array}$ \\
\hline umbeliferone (36) & P. riedelianus & Antioxidant & $\begin{array}{l}\text { Hoult, Paya, } 1996 \\
\text { Guerreiro et al., } 2001\end{array}$ \\
\hline $\begin{array}{l}\text { 13,13-dimethyl-6 methoxy-2H, } \\
8 H \text {-pyrano- }(2,3) \text {-cromen-2-one (37) }\end{array}$ & P. riedelianus & - & Guerreiro et al., 2001 \\
\hline $\begin{array}{l}\text { 7-hydroxy-3-(1', 1'-dimethyl-allyl)- } \\
\text { 8-methoxy-coumarin (38) }\end{array}$ & P. riedelianus & $\begin{array}{l}\text { Bactericide } \\
\text { Antifungic }\end{array}$ & Guerreiro et al., 2001 \\
\hline 3'-hydroxy-dipetalactone (39) & P. grandiflorus & - & De Souza et al., 2001 \\
\hline $\begin{array}{l}\text { 3',4'-dihydro-3'-hydroxy- } \\
\text { dipetalactone (40) }\end{array}$ & P. grandiflorus & Antifungic & De Souza et al., 2003 \\
\hline 3-(1',1'-dimethyl-allyl)-scopoletin (41) & P. grandiflorus & Antifungic & De Souza et al., 2003 \\
\hline
\end{tabular}<smiles>CC1(C)C=Cc2cc3ccc(=O)oc3cc2O1</smiles>

(15)<smiles>COc1c2c(cc3oc(=O)ccc13)OC(C)(C)C=C2</smiles>

(16)<smiles>COc1c2c(cc3ccc(=O)oc13)C=CC(C)(C)O2</smiles>

(17)<smiles>CC(C)=CCOc1c2c(cc3ccc(=O)oc13)C=CC(C)(C)O2</smiles><smiles>CC1(C)C=Cc2c(O)cc3oc(=O)ccc3c2O1</smiles>

(19)<smiles>COc1c2ccoc2cc2oc(=O)ccc12</smiles>

(20)<smiles>COc1c2occc2cc2ccc(=O)oc12</smiles>

(21)<smiles>COc1c2occc2c(C)c2ccc(=O)oc12</smiles>

(22)<smiles>CC(C)=CCOc1c2occc2cc2ccc(=O)oc12</smiles>

(23)<smiles>COCc1c2occc2cc2ccc(=O)oc12</smiles>

(24)<smiles>C=C(C)C1Cc2c(cc3oc(=O)ccc3c2O)O1</smiles>

(25)<smiles>COc1cc2cc(OC)c3occc3c2oc1=O</smiles>

(26)<smiles>COc1cc2ccc3occc3c2oc1=O</smiles>

(27)<smiles>COc1cc2ccc(=O)oc2cc1OC</smiles>

(28)<smiles>COc1c2ccoc2c(C)c2oc(=O)ccc12</smiles>

(29)<smiles>COc1ccc2ccc(=O)oc2c1CC=C(C)C</smiles>

FIGURE 2 - Chemical structures of some coumarins isolated from de Pilocarpus species. 
<smiles>COc1ccc2ccc(=O)oc2c1C[C@H](O)C(C)(C)O</smiles><smiles>COc1cc2ccc(=O)oc2c2ccoc12</smiles>

(32)<smiles>CC(C)=CCC/C(C)=C/COc1ccc2ccc(=O)oc2c1</smiles>

(33)

(31)<smiles>C=CC(C)(C)c1cc2cc3c(cc2oc1=O)OC(C(C)(C)O)C3</smiles>

(34)<smiles>COc1ccc2c(c1)C=C1CCC(C)(C)OC1O2</smiles>

(37)<smiles>CC1(C)C=Cc2c(ccc3ccc(=O)oc23)O1</smiles>

(35)<smiles>C=CC(C)(C)C1Cc2cc3cc(O)oc3c(OC)c2OC1=O</smiles><smiles>O=c1ccc2ccc(O)cc2o1</smiles>

(36)<smiles>CC1(C)C=Cc2c3c(c4ccc(=O)oc4c2O1)OC(C)(C)C(C)(O)C=C3</smiles><smiles>CC1(C)C=Cc2c(c3c(c4ccc(=O)oc24)OC(C)(C)[C@@H](O)C3)O1</smiles>

(40)<smiles>C=CC(C)(C)C1Cc2c(c(C)c3occc3c2OC)OC1=O</smiles>

(41)

FIGURE 2 - Chemical structures of some coumarins isolated from de Pilocarpus species (continuation).

TABLE V - Flavonoids isolated from Pilocarpus species and some related biological activities

\begin{tabular}{|c|c|c|c|}
\hline Flavonoid & Species & Activity & References \\
\hline $\begin{array}{l}\text { 3,4',5,7-tetrahydroxy-3',6-dimethoxy- } \\
\text { flavone-3-O- } \beta \text {-rutinoside (42) }\end{array}$ & P. trachyllophus & & Bertrand et al., 2001 \\
\hline $\begin{array}{l}\text { 3,4',5,7-tetrahydroxy-6-methoxy- } \\
\text { flavone-3-O- } \beta \text {-rutinoside (43) }\end{array}$ & P. trachyllophus & & Bertrand et al., 2001 \\
\hline patulentine-3- $O$ - $\beta$-rutinoside (44) & P. trachyllophus & $\begin{array}{l}\text { Antioxidant } \\
\text { (aglicone) }\end{array}$ & Bertrand et al., 2001 \\
\hline axilarin (45) & P. trachyllophus & Antioxidant & $\begin{array}{l}\text { Bertrand et al., } 2001 \\
\text { Kim et al., } 2002\end{array}$ \\
\hline hesperidin (46) & P. trachyllophus & $\begin{array}{l}\text { Antioxidant } \\
\text { Sedative }\end{array}$ & $\begin{array}{l}\text { Andrade Neto et al., } 1996 \\
\text { Kim } \text { et al., } 2002\end{array}$ \\
\hline
\end{tabular}


<smiles>[R]c1cc(-c2oc3cc(O)c(OC)c(O)c3c(=O)c2[R2])ccc1O</smiles>

(42) $\mathrm{R} 1=\mathrm{OCH}_{3}$ R2 $=3-O-\beta-D-$ rutinosil

(43) $\mathrm{R} 1=\mathrm{H}$ R2 = 3-O- $\beta-D-$ rutinosil<smiles>[R]c1cc(O)c2c(c1)O[C](c1ccc(OC)c(O)c1)CC2=O</smiles>

(46) $R=7-O-\beta$-L- rutinosil<smiles>[R20]c1c(-c2ccc(O)c(O)c2)oc2cc(O)c(OC)c(O)c2c1=O</smiles>

(44) $\mathrm{R} 1=3-\mathrm{O}-\beta-\mathrm{D}-$-rutinosil

(45) $\mathrm{R} 1=\mathrm{CH}_{3}$



D-rutinosil

FIGURE 3 - Chemical structures of flavonoids isolated from Pilocarpus species.

through the mevalonate pathway that gives rise to the mono- (C10), sesqui- (C15), di- (C20) and triterpenes (C30).

There are many references about the importance of terpenes in plant metabolism, mainly as essential oils. Although it is of general sense that terpenes represent a fundamental tool in pollinator's attraction, guaranteeing a species reproduction. They also displayed a variety of biologic activities such as insecticide, antimicrobial and antiseptic (Figueiredo, 1992). The essential oils of $P$. spicatus, $P$. gradiflorus and $P$. pauciflorus species exhibited bactericidal activity against Bacilus subtilis, Salmonella typhimurium, Escherichia coli and Pseudomonas aeruginosa (Santos et al., 1997).

Although the occurrence of triterpenes and steroids in plants is quite common, and these compounds were only reported in three Pilocarpus species (Table VI and Figure 4). For $P$. grandiflorus the triterpenes b-amyrin (109), occotilone (111) and germanicol (114) were described. The b-amyrin acetate (110) obtained from $P$. trachyllophus showed a high cytostatic activity against cells of HEp-2 (Gomez et al., 1997).

Additional pharmacological activities, such as angiogenic, antihelminthic, antimicrobial and antitumoral, were described only for the steroid $\beta$-sitosterol (86) (Moon et al., 1999; Villasenor et al., 2002; Singh, Singh, 2003; Awad et al., 2003).

\section{Other secondary metabolites isolated fom Pilocarpus species}

Besides alkaloids, coumarins, flavonoids and terpenes there are minor compounds that can occur in some Pilocarpus species. Among these compounds are lignans, hydrocarbons and its alcohols, amides, carboxilic acids, esters and ketones derivatives (Table VII and Figure $5)$.

Lignans (also $L$-phenylalanine derivatives) were obtained from two different Pilocarpus species (Table VII). Lignans play an important role in the plant sustentation as cell wall constituents. The lignan lirioresinol B (124), isolated from $P$. grandiflorus (De Souza et al., 2003), showed antichagasic activity (Sauvain et al., 1996).

The amide pellitorine (125) was found in $P$. trachyllophus (Andrade Neto et al., 1995). This substance is frequently isolated from Piper species and it is widely known as an insecticide (He et al., 2002). 
TABLE VI - Terpenoids isolated from Pilocarpus species

\begin{tabular}{|c|c|c|}
\hline Terpene (class) & Species & References \\
\hline \multicolumn{3}{|l|}{ Monoterpenes } \\
\hline $\begin{array}{l}\text { 3,7.7-trimethylbicyclo[3.1.1]- } \\
\text { 2-heptene (47) }\end{array}$ & P. microphyllus, P. affinis & Craveiro et al., 1979 \\
\hline$\alpha$-pinene (48) & $\begin{array}{l}\text { P. jaborandi, } P \text {. microphyllus, } \\
\text { P. affinis, } P \text {. spicatus, } \\
\text { P. grandiflorus }\end{array}$ & $\begin{array}{l}\text { Craveiro et al., } 1979 \\
\text { Santos et al., } 1997 \\
\text { Andrade Neto et al., } 2002\end{array}$ \\
\hline$\beta$-pinene (49) & P. spicatus, $P$. grandiflorus & $\begin{array}{l}\text { Santos et al., } 1997 \\
\text { Andrade Neto et al., } 2002\end{array}$ \\
\hline canfene (50) & P. spicatus & Andrade Neto et al., 2002 \\
\hline borneol (51) & P. spicatus & Andrade Neto et al., 2002 \\
\hline linalool (52) & P. spicatus, $P$. grandiflorus & $\begin{array}{l}\text { Santos et al., } 1997 \\
\text { Andrade Neto et al., } 2002\end{array}$ \\
\hline mircene (53) & $\begin{array}{l}\text { P. jaborandi, P. affinis, P. spicatus, } \\
\text { P. grandiflorus }\end{array}$ & $\begin{array}{l}\text { Craveiro et al., } 1979 \\
\text { Santos et al., } 1997 \\
\text { Andrade Neto et al., } 2002\end{array}$ \\
\hline$E$ - $\beta$-ocimene (54) & P. spicatus, $P$. pennatifolius & $\begin{array}{l}\text { Andrade Neto et al., } 2002 \\
\text { Santos et al., } 2004\end{array}$ \\
\hline$Z$ - $\beta$-ocimene (55) & P. pennatifolius & Santos et al., 2004 \\
\hline Limonene (56) & $\begin{array}{l}\text { P. jaborandi, microphyllus, } P \text {. affinis, } \\
\text { P. trachyllophus, P. spicatus, } \\
\text { P. grandiflorus }\end{array}$ & $\begin{array}{l}\text { Craveiro et al., } 1979 \\
\text { Andrade Neto et al., } 1995 \\
\text { Santos et al., } 1997 \\
\text { Andrade Neto et al., 2000, } 2002\end{array}$ \\
\hline Sabinene (57) & P. spicatus & Andrade Neto et al., 2002 \\
\hline$\delta$-3-carene (58) & P. spicatus & Andrade Neto et al., 2002 \\
\hline$\alpha$-terpinene (59) & P. spicatus & Andrade Neto et al., 2002 \\
\hline$\gamma$-terpinene (60) & P. spicatus & Andrade Neto et al., 2002 \\
\hline$\alpha$-terpineol (61) & P. spicatus & Andrade Neto et al., 2002 \\
\hline terpinen-4-ol (62) & P. spicatus & Andrade Neto et al., 2002 \\
\hline$p$-cimene (63) & P. spicatus & Andrade Neto et al., 2002 \\
\hline$\beta$-felandrene (64) & P. spicatus & Andrade Neto et al., 2002 \\
\hline 1,8-cineole (65) & P. spicatus & Andrade Neto et al., 2002 \\
\hline \multicolumn{3}{|l|}{ Sesquiterpenes } \\
\hline$\overline{Z, Z \text {-farnesol (66) }}$ & P. spicatus & Andrade Neto et al., 2002 \\
\hline$E$-nerolidol (67) & P. spicatus & Andrade Neto et al., 2002 \\
\hline$\beta$-bisabolene (68) & P. trachyllophus, P. spicatus & $\begin{array}{l}\text { Andrade Neto et al., } 1995 \\
\text { Santos et al., } 1997 \\
\text { Andrade Neto et al., 2000, } 2002\end{array}$ \\
\hline$\delta$-cadinene (69) & $\begin{array}{l}\text { P. microphyllus, P. trachyllophus, } \\
\text { P. spicatus. P. pennatifolius }\end{array}$ & $\begin{array}{l}\text { Craveiro et al., } 1979 \\
\text { Andrade Neto et al., } 1995 \\
\text { Andrade Neto et al., 2000, } 2002 \\
\text { Santos et al., } 2004\end{array}$ \\
\hline$\gamma$-cadinene (70) & P. trachyllophus, P. spicatus & $\begin{array}{l}\text { Andrade Neto et al., } 1995 \\
\text { Santos et al., } 1997 \\
\text { Andrade Neto et } \text { al., 2000, } 2002\end{array}$ \\
\hline germacrene D (71) & P. trachyllophus, P. spicatus & $\begin{array}{l}\text { Andrade Neto et al., } 1995 \\
\text { Andrade Neto et al., } 2002\end{array}$ \\
\hline
\end{tabular}


TABLE VI - Terpenoids isolated from Pilocarpus species (continuation)

\begin{tabular}{|c|c|c|}
\hline Terpene (class) & Species & References \\
\hline \multicolumn{3}{|l|}{ Sesquiterpenes } \\
\hline$\beta$-cubebene (72) & P. spicatus, $P$. pennatifolius & $\begin{array}{l}\text { Andrade Neto et al., } 1995 \\
\text { Andrade Neto et al., } 2002 \\
\text { Santos et al., } 2004\end{array}$ \\
\hline$\gamma$-muurolene (73) & P. spicatus, $P$. pennatifolius & $\begin{array}{l}\text { Andrade Neto et al., } 1995 \\
\text { Andrade Neto et al., } 2002 \\
\text { Santos } \text { et al., } 2004\end{array}$ \\
\hline$\alpha$-muurolene (74) & P. spicatus & Andrade Neto et al., 2002 \\
\hline germacrene-4-D-ol (75) & P. spicatus & Andrade Neto et al., 2002 \\
\hline$\alpha$-cadinol (76) & P. spicatus & Andrade Neto et al., 2002 \\
\hline cubenol (77) & P. spicatus & Andrade Neto et al., 2002 \\
\hline T-muurolol (78) & P. spicatus & Andrade Neto et al., 2002 \\
\hline calacorene (79) & P. riedelianus & Guerreiro et al., 2001 \\
\hline$\alpha$-calacorene (80) & P. riedelianus & Guerreiro eta., 2001 \\
\hline$\gamma$-calacorene (81) & P. riedelianus & Guerreiro et al., 2001 \\
\hline cadalene (82) & P. riedelianus & Guerreiro et al., 2001 \\
\hline sesquichamaenol (83) & P. riedelianus & Guerreiro et al., 2001 \\
\hline sesquichamaenol A (84) & P. riedelianus & Guerreiro et al., 2001 \\
\hline$\delta$-elemene (85) & P. spicatus & Andrade Neto et al., 2002 \\
\hline germacrene A (86) & P. spicatus & Andrade Neto et al., 2002 \\
\hline germacrene B (87) & P. pennatifolius & Santos et al., 2004 \\
\hline selinene (88) & P. affinis, $P$. pennatifolius & $\begin{array}{l}\text { Craveiro et al., } 1979 \\
\text { Santos et al., } 2004\end{array}$ \\
\hline$\beta$-elemene (89) & P. spicatus, $P$. pennatifolius & $\begin{array}{l}\text { Andrade Neto et al., } 1995 \\
\text { Andrade Neto et al., } 2002 \\
\text { Santos et al., } 2004\end{array}$ \\
\hline bicyclogermacrene (90) & P. spicatus, P. pennatifolius & $\begin{array}{l}\text { Andrade Neto et al., } 2002 \\
\text { Santos et al., } 2004\end{array}$ \\
\hline elemol (91) & P. spicatus & Andrade Neto et al., 2002 \\
\hline$\beta$-eudesmol (92) & P. spicatus & Andrade Neto et al., 2002 \\
\hline cyclosativene (93) & P. spicatus & Andrade Neto et al., 2002 \\
\hline caryophyllene oxide (94) & P. trachyllophus, $P$. spicatus & $\begin{array}{l}\text { Andrade Neto et al., } 1995 \\
\text { Santos et al., } 1997 \\
\text { Andrade Neto et al., 2000, } 2002\end{array}$ \\
\hline$\alpha$-humulene (95) & $\begin{array}{l}\text { P. microphyllus, P. trachyllophus, } \\
\text { P. spicatus, P. grandiflorus, } \\
\text { P. pennatifolius }\end{array}$ & $\begin{array}{l}\text { Craveiro et al., } 1979 \\
\text { Andrade Neto et al., } 1995 \\
\text { Santos et al., } 1997 \\
\text { Andrade Neto et al., 2000, } 2002 \\
\text { Santos et al., } 2004\end{array}$ \\
\hline$\alpha$-copaene (96) & $\begin{array}{l}\text { P. microphyllus, P. spicatus, } \\
\text { P. pennatifolius }\end{array}$ & $\begin{array}{l}\text { Craveiro et al., } 1979 \\
\text { Andrade Neto et al., } 2002 \\
\text { Santos et al., } 2004\end{array}$ \\
\hline$\beta$-borbonene (97) & P. trachyllophus, $P$. pennatifolius & $\begin{array}{l}\text { Andrade Neto et al., } 1995 \\
\text { Andrade Neto et al., } 2002 \\
\text { Santos et al., } 2004\end{array}$ \\
\hline$\beta$-caryophyllene (98) & $\begin{array}{l}\text { P. microphyllus, P. trachyllophus, } \\
\text { P. spicatus, P. pennatifolius }\end{array}$ & $\begin{array}{l}\text { Craveiro et al., } 1979 \\
\text { Andrade Neto et al., } 1995 \\
\text { Santos et al., } 1997 \\
\text { Andrade Neto et al., 2000, } 2002 \\
\text { Santos et al., } 2004\end{array}$ \\
\hline
\end{tabular}


TABLE VI - Terpenoids isolated from Pilocarpus species (continuation)

\begin{tabular}{|c|c|c|}
\hline Terpene (class) & Species & References \\
\hline \multicolumn{3}{|l|}{ Sesquiterpenes } \\
\hline $\begin{array}{l}\alpha \text {-guaiaene (99) } \\
\text { spathulenol (100) }\end{array}$ & $\begin{array}{l}\text { P. microphyllus } \\
\text { P. trachyllophus, P. pennatifolius }\end{array}$ & $\begin{array}{l}\text { Craveiro et al., } 1979 \\
\text { Andrade Neto et al., } 1995 \\
\text { Andrade Neto et al., } 2000 \\
\text { Santos } \text { et al., } 2004\end{array}$ \\
\hline$\alpha$-cubebene (101) & P. spicatus & Andrade Neto et al., 2002 \\
\hline allo-aromadendrene (102) & P. spicatus & Andrade Neto et al., 2002 \\
\hline globulol (103) & P. spicatus & Andrade Neto et al., 2002 \\
\hline viridiflorol (104) & P. spicatus & Andrade Neto et al., 2002 \\
\hline guaiol (105) & P. spicatus & Andrade Neto et al., 2002 \\
\hline bulnesol (106) & P. spicatus & Andrade Neto et al., 2002 \\
\hline \multicolumn{3}{|l|}{ Diterpenes } \\
\hline sandaracopimaradiene (107) & P. jaborandi & Craveiro et al., 1979 \\
\hline angustifolin (108) & P. riedelianus & Guerreiro et al., 2001 \\
\hline \multicolumn{3}{|l|}{ Triterpenes } \\
\hline$\beta$-amyrin (109) & P. grandiflorus & De Souza et al., 2003 \\
\hline$\beta$-amyrin acetate (110) & P. trachyllophus & Bertrand et al., 2001 \\
\hline ocotillone (111) & P. grandiflorus & De Souza et al., 2003 \\
\hline $\begin{array}{l}\text { 3- } \beta \text {-acetoxy-24-methyl-25-ethyl- } \\
\text { 20,24-epoxydammarane (112) }\end{array}$ & P. spicatus & Andrade Neto et al., 1994 \\
\hline $\begin{array}{l}\text { 3-oxo-24-methyl-25-ethyl- } \\
\text { 20,24-epoxydammarane (113) }\end{array}$ & P. spicatus & Andrade Neto et al., 1994 \\
\hline germanicol (114) & P. grandiflorus & De Souza et al., 2003 \\
\hline \multicolumn{3}{|l|}{ Steroids } \\
\hline$\beta$-sitosterol (115) & P. grandiflorus & De Souza et al., 2003 \\
\hline$\beta$-sitosterone (116) & P. grandiflorus & De Souza et al., 2003 \\
\hline 7-oxo- $\beta$-sitosterol (117) & P. grandiflorus & De Souza et al., 2003 \\
\hline stigmast-3,7-dione (118) & P. grandiflorus & De Souza et al., 2003 \\
\hline 7- $\beta$-hydroxy-stigmast-4-en-3-one (119) & P. grandiflorus & De Souza et al., 2003 \\
\hline \multicolumn{3}{|l|}{ Polyprenols } \\
\hline 12-prenol (120) & P. trachyllophus & Bertrand et al., 2001 \\
\hline$[3 E, 9 Z]-13$-prenol (121) & P. trachyllophus & Bertrand et al., 2001 \\
\hline$[3 E, 10 Z]$-14-prenol (122) & P. trachyllophus & Bertrand et al., 2001 \\
\hline
\end{tabular}

The pattern of $n$-alkanes is considered an important taxonomic marker in plant classification accordingly to Eglinton et al. (1962). The $n$-alkanes identified in leaf epicuticular waxes of different Pilocarpus species with a carbon chain ranging from $\mathrm{C}_{14}$ to $\mathrm{C}_{33}$ (Skorupa et al., 1998). An aromatic hydrocarbon $\left(\mathrm{C}_{20}\right)$ was detected only in $P$. jaborandi and this substance was considered a marker for the species (Negri et al., 1998).

\section{CONCLUDING REMARKS}

In this review, information concerning the occurrence and pharmacological activities of secondary metabolites isolated from Pilocarpus spp were collected. Alkaloids, coumarins, flavonoids and terpenoids are current compounds to this genus (Guerreiro et al., 2001). Compounds derived from $L$-phenylalanine are predominant in this genus. 


\section{Monoterpenes}<smiles>CC1=CC2CCC1C2</smiles>

(47)

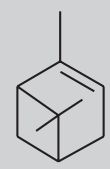

(48)

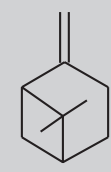

(49)<smiles>C=C1C2CCC(C2)C1(C)C</smiles>

(50)<smiles>CC1(C)C2CCC1(C)C(O)C2</smiles>

(51)<smiles>C=CC(C)(O)CCC=C(C)C</smiles>

(52)<smiles>C=CC(=C)CCC=C(C)C</smiles>

(53)<smiles>C=C/C(C)=C\CC=C(C)C</smiles>

(54)<smiles>C=C/C(C)=C/CC=C(C)C</smiles>

(55)<smiles>C=C(C)C1CC=C(C)CC1</smiles>

(56)<smiles>C=C1CCC(C(C)C)C1</smiles>

(57)<smiles>CC1=CCC2C(C1)C2(C)C</smiles>

(58)<smiles>CC1=CC=C(C(C)C)CC1</smiles>

(59)<smiles>CC1=CCC(C(C)C)=CC1</smiles>

(60)<smiles>CC1=CCC(C(C)(C)O)CC1</smiles>

(61)<smiles>CC1=CCC(O)(C(C)C)CC1</smiles>

(62)<smiles>Cc1ccc(C(C)C)cc1</smiles>

(63)<smiles>CC1=CCC(C(C)C)C=C1</smiles>

(64)

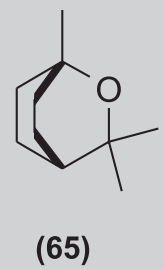

(65)

FIGURE 4 - Chemical structures of some terpenoids isolated from Pilocarpus species.

Although the medicinal value of pilocarpine is widely recognized, but the importance of other secondary metabolites obtained from Pilocarpus species was not yet well described. The summary of the main bioactive compounds, other than pilocarpine, described for Pilocarpus species are listed below:

1. From the 16 known Pilocarpus species, alkaloids have only been described in eight species (almost 1 alkaloid/ species) (Table III), and for the majority of them the bioactivity was not yet determined;
2. There are a few reports over the presence of coumarins in Pilocarpus spp., however the pharmacological activities of this class of compounds are numerous;

3. Flavonoids have been increasingly described as antioxidant drugs but these compounds were only found in P. trachyllophus (Table V);

4. Only two lignans were isolated from two Pilocarpus species and one of them has been described as an antichagasic agent (Table VII).

The use of plants in folk medicine has lead scientists 


\section{Sesquiterpenes}<smiles>CC(C)=CCC/C=C(/C)CC/C(C)=C\C(=O)O</smiles>

(66)<smiles>C=C[C@](C)(O)CCC=C(C)CCC=C(C)C</smiles>

(67)<smiles>C=C(CCC=C(C)C)[C@H]1CC=C(C)CC1</smiles>

(68)<smiles>CC1=C[C@H]2C(=C(C)CC[C@@H]2C(C)C)CC1</smiles>

(69)<smiles>C=C1CC[C@H](C(C)C)[C@H]2C=C(C)CC[C@@H]12</smiles>

(70)<smiles>C=C1C=CC(C(C)C)CCC(C)=CCC1</smiles>

(71)<smiles>CC1=CCC23C(C)CC[C@@H](C(C)C)[C@H]2[C@H]13</smiles>

(72)<smiles>C=C1CC[C@H](C(C)C)[C@H]2C=C(C)CC[C@H]12</smiles>

(73)<smiles>CC[C@H]1C=C(C)CCC/C(C)=C\[C@@H]1C(C)C</smiles>

(74)<smiles>C=C(C)CC(O)C=C(C)CC[C@@H](/C=C/C(=C)CC)C(C)C</smiles>

(75)<smiles>CC1=C[C@H]2C(CC1)[C@H](C)CC[C@H]2C(C)C</smiles>

(76)

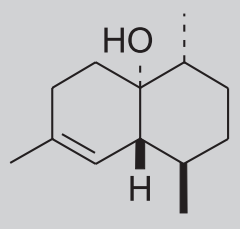

(77)<smiles>C/C1=C/[C@H](C)CC[C@H](O)[C@H](C)CC1</smiles>

(78)<smiles>Cc1ccc2c(c1)C(C(C)C)CCC2C</smiles>

(79)<smiles>CC1=CCC(C(C)C)c2cc(C)ccc21</smiles>

(80)<smiles>Cc1ccc2c(c1)C(C(C)C)=CCC2C</smiles>

(81)<smiles>C=C(C)c1ccccc1C(=C)C(C)C</smiles>

(82)<smiles>C=C(C)CCC(c1ccccc1O)C(C)C</smiles>

(83)<smiles>CC(C)/C=C/C(c1ccccc1O)C(C)C</smiles>

(84)

FIGURE 4 - Chemical structures of some terpenoids isolated from Pilocarpus species (continuation). 


\section{Sesquiterpenes}<smiles>C=C[C@]1(C)CCC(C(C)C)=CC1C(=C)C</smiles>

(85)<smiles>CC1=CC2C(CCC=C(C)C2(C)C)CCC1</smiles>

(90)

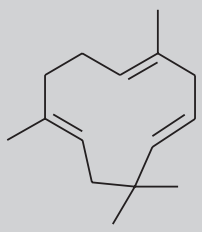

(95)

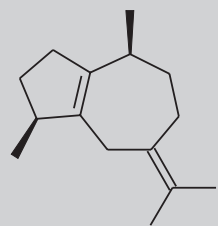

(99)

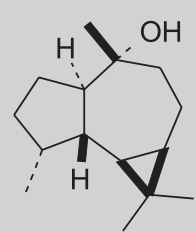

(103)

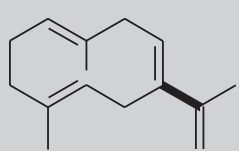

(86)<smiles>C=C[C@]1(C)CCC(C(C)(C)O)CC1C(=C)C</smiles>

(91)<smiles>CC(C)=C1CCC2=CCCC(C)=C2C1</smiles>

(87)<smiles>C=C1CCC[C@]2(C)CC[C@@H](C(=C)C)C[C@H]12</smiles>

(88)

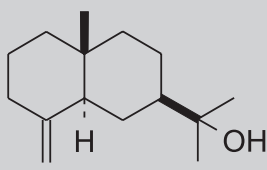

(92)



(93)

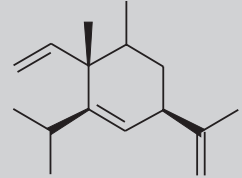

(89)

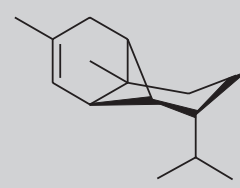

(96)

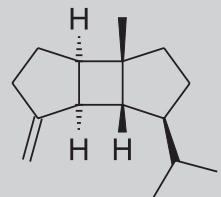

(97)

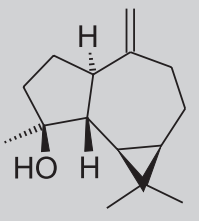

(100)

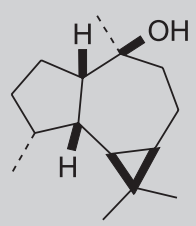

(104)

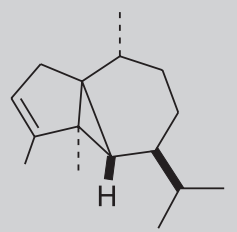

(101)<smiles>CC1CC[C@H](C(C)(C)O)CC2=C1CCC2C</smiles>

(105)

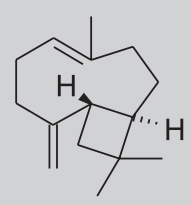

(98)

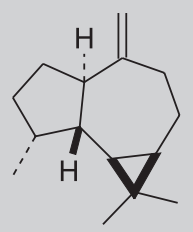

(102)

\section{Diterpenes}
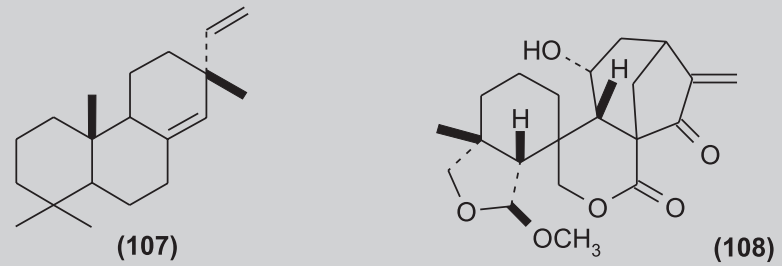

FIGURE 4 - Chemical structures of some terpenoids isolated from Pilocarpus species (continuation). 
Triterpenes

<smiles>CC(=O)O[C@H]1CC[C@@]2(C)C(CC[C@@]3(C)C2CCC2=C4CC(C)(C)CC[C@]4(C)CC[C@H]23)C1(C)C</smiles><smiles>CC1(C)C(=O)CC[C@@]2(C)C1CCC1[C@](C)([C@H]3CC[C@@H](C(C)(C)O)O3)CC[C@]12C</smiles>

(111)

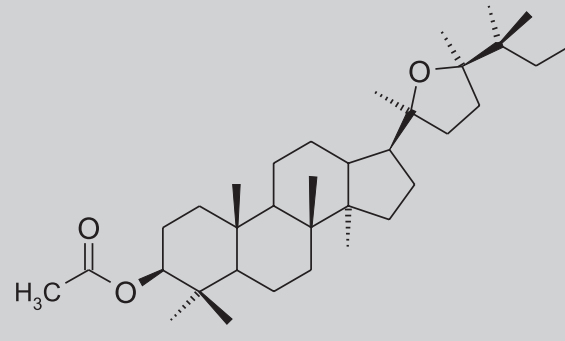

(112)

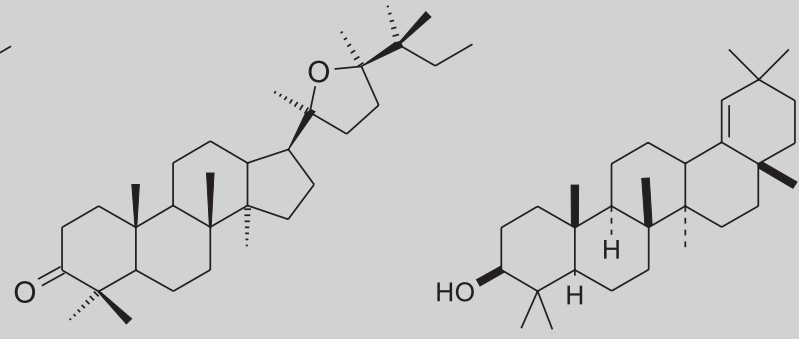

(113)

(114)

\section{Steroids}<smiles>CCC(CCCC(C)[C@H]1CC[C@H]2[C@@H]3CC=C4CC(O)CC[C@]4(C)C3CC[C@@]21C)C(C)C</smiles><smiles>CCC(CCCC(C)[C@H]1CCC2C3CC=C4CC(=O)CC[C@]4(C)C3CC[C@]21C)C(C)C</smiles><smiles>CCC(CCCC(C)[C@H]1CC[C@H]2C3C(=O)C=C4CC(O)CC[C@]4(C)[C@H]3CC[C@@]21C)C(C)C</smiles><smiles>CCC(/C=C\C(C)[C@H]1CC[C@H]2C(=O)C=C3CC(=O)CC[C@]3(C)[C@H]2[C@H]1C)C(C)C</smiles><smiles>CCC(/C=C\C(C)[C@H]1CC[C@H]2[C@H]3C(O)CC4=CC(=O)CC[C@@]4(C)[C@H]3CC[C@@]21C)C(C)C</smiles>

Prenols

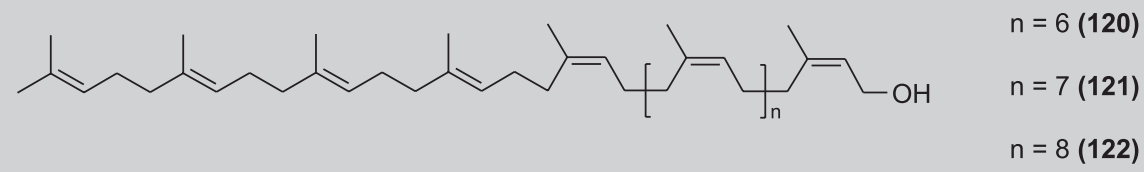

FIGURE 4 - Chemical structures of some terpenoids isolated from Pilocarpus species (continuation). 
TABLE VII - Miscellaneous secondary metabolites isolated from Pilocarpus species and their respective biological activities

\begin{tabular}{|c|c|c|c|}
\hline Compound & Species & Activity & References \\
\hline (-)-episesamin (123) & P. trachyllophus & _- & Andrade Neto et al., 1995 \\
\hline lirioresinol B (124) & P. grandiflorus & Antichagásica & $\begin{array}{l}\text { De Souza et al., } 2003 \\
\text { Sauvain et al., } 1996\end{array}$ \\
\hline pellitorine (125) & P. trachyllophus & Inseticida & $\begin{array}{l}\text { Andrade Neto et al., } 1995 \\
\text { He et al., } 2002\end{array}$ \\
\hline eugenol (126) & P. pennatifolius & $\begin{array}{l}\text { Inseticida } \\
\text { Anestésica } \\
\text { Antioxidante } \\
\text { Antifúngica }\end{array}$ & $\begin{array}{l}\text { Santos et al., } 2004 \\
\text { Park et al.,2000 } \\
\text { Ogata et al., } 2000 \\
\text { Priyadarsini et al., } 1998 \\
\text { Meepagala et al., } 2002\end{array}$ \\
\hline$N^{\prime}, N^{\prime}$-dimethyl antranilic acid (127) & P. grandiflorus & - & De Souza et al., 2003 \\
\hline vanilic acid (128) & P. grandiflorus & - & De Souza et al., 2003 \\
\hline siringaldehyde (129) & P. grandiflorus & - & De Souza et al., 2003 \\
\hline 2-undecanone (130) & $\begin{array}{l}\text { P. spicatus } \\
\text { P.trachyllophus } \\
\text { P. jaborandi }\end{array}$ & - & $\begin{array}{l}\text { Santos et al., } 1997 \\
\text { Andrade Neto et al., } 1995 \\
\text { Andrade Neto et al., 2000, } 2002\end{array}$ \\
\hline 2-tridecanone (131) & $\begin{array}{l}\text { P. spicatus } \\
\text { P.trachyllophus } \\
\text { P. jaborandi }\end{array}$ & - & $\begin{array}{l}\text { Santos et al., } 1997 \\
\text { Andrade Neto et al., } 1995 \\
\text { Andrade Neto et al., 2000, } 2002\end{array}$ \\
\hline 1-hydroxy-2-tridecanone (132) & P. trachyllophus & - & Andrade Neto et al., 1996 \\
\hline 2-hydroxy-ethyl-glutarate (133) & P. racemosus & - & Compagnone \& Rodriguez, 1993 \\
\hline methyl hexadecanoate (134) & P. pennatifolius & - & Santos et al., 2004 \\
\hline hexanol (135) & P. pennatifolius & - & Santos et al., 2004 \\
\hline undecanol (136) & P. pennatifolius & - & Santos et al., 2004 \\
\hline $\begin{array}{l}\text { 1-phenyl-5-vinyl-5,9-dimethyl- } \\
\text { decane (137) }\end{array}$ & P. jaborandi & - & $\begin{array}{l}\text { Negri et al., } 1998 \\
\text { Skorupa et al., } 1998\end{array}$ \\
\hline tridecane (138) & P. pennatifolius & - & Santos et al., 2004 \\
\hline pentadecane (139) & P. pennatifolius & - & Santos et al., 2004 \\
\hline$n$-alcanes $(\mathbf{1 4 0})$ & $\begin{array}{l}\text { P. alatus, } \\
\text { P. carajaensis, } \\
\text { P. giganteus, } \\
\text { P. sulcatus, } \\
\text { P. pauciflorus, } \\
\text { P. jaborandi, } \\
\text { P. grandiflorus, } \\
\text { P. riedelianus, } \\
\text { P. microphyllus }\end{array}$ & - & Skorupa et al., 1998 \\
\hline
\end{tabular}

to find a wide variety of active substances of invaluable importance to mankind. Considering the variety of metabolites isolated from Pilocarpus, these species represent a vast research field to be exploited.

\section{RESUMO}

\section{Pilocarpus spp.: Revisão sobre sua constituição química e atividades biológicas}

Espécies de Pilocarpus têm sido exploradas como única fonte do alcalóide imidazólico pilocarpina (utilizado no tratamento do glaucoma) desde o isolamento dessa substância até os dias atuais. A maioria das espécies de Pilocarpus conhecida é nativa do Brasil e, devido à importância medicinal que a pilocarpina possui e ao desmatamento, várias se encontram em risco de extinção. Outros metabólitos secundários entre os quais cumarinas, flavonóides e terpenos foram descritos em espécies desse gênero. Nesta revisão foram relacionados os metabólitos secundários isolados em diversas espécies de Pilocarpus bem como suas atividades biológicas. Apesar da varieda- 


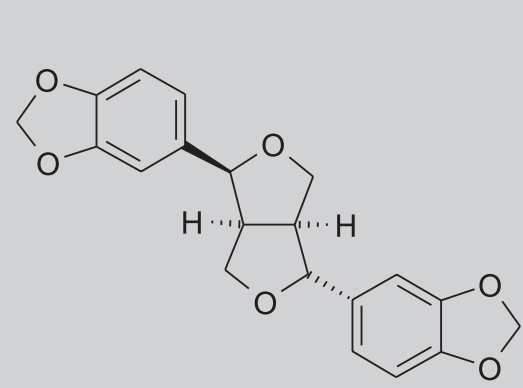

(123)<smiles>COc1cc([C@@H]2OC[C@H]3[C@H]2CO[C@H]3c2cc(OC)c(O)c(OC)c2)cc(OC)c1O</smiles><smiles>CN(C)c1ccccc1C(=O)O</smiles>

(127)<smiles>O=C(O)c1ccc(O)c(O)c1</smiles>

(128)<smiles>COc1cc(C=O)cc(OC)c1OC</smiles>

(129)<smiles>CCCC(C)C(C)=O</smiles><smiles>CCCC(C)C(=O)OC</smiles>

$$
n=7 \text { (134) }
$$<smiles>CCCCCCO</smiles>

(135)<smiles>CCCCCCCCCCCO</smiles>

(136)<smiles>C=CC(C)(CCCCc1ccccc1)CCCC(C)C</smiles>

(137)

$$
\begin{aligned}
n=5(138) \\
n=6(139)
\end{aligned} \mathrm{CH}_{3}
$$

$$
n=6-15(140)
$$

FIGURE 5 - Chemical structures of miscellaneous secondary metabolites isolated from Pilocarpus species. 
de de estruturas e as importantes atividades biológicas já descritas na literatura para as outras classes de metabólitos secundários, ainda há um vasto campo de estudo para as espécies de Pilocarpus.

UNITERMOS: Rutaceae. Pilocarpus spp. Metabólitos secundários. Atividades biológicas.

\section{REFERENCES}

ALBUQUERQUE, B.W.P. Rutaceae do Estado da Guanabara. An. Acad. Bras. Ciênc., v. 40, n. 4, p. 500, 1968. Apud: ALBUQUERQUE, B.W.P. Rutaceae. In.: RIZZO J.A.,org. Flora do Estado de Goiás. Goiânia: Editora da UFG, 1985. v. 6. 35 p.

ALBUQUERQUE, B.W.P. Rutaceae. In: RIZZO J.A.,org. Flora do Estado de Goiás. Goiânia: Editora da UFG, 1985. v. 6.35 p.

AMARO-LUIS, J.M.; MASSANET, G.M.; PANDO, E.; RODRIGUEZ LUIZ, F. New coumarins from Pilocarpus goudotianus. Planta Med., v. 56, p. 304-306, 1990.

ANDRADE NETO, M.; SILVEIRA, E.R.; BRAZ-FILHO, R.; GAMBARDELA, M.T.P.; SANTOS, R.H.A. 24methyl-25-ethyldammarane derivatives from Pilocarpus spicatus. Phytochemistry, v. 35, n. 3, p. 739-743, 1994.

ANDRADE NETO, M.; MAFEZOLI, J.; CUNHA, U.A.; SILVEIRA, S.C.; SILVEIRA, E.R. Volatile constituents of Pilocarpus trachyllophus Holmes. J. Essent. Oil Res., v. 7, p. 671-674, 1995.

ANDRADE NETO, M.; MENDES, P.H.; SILVEIRA, E.R. An imidazole alkaloid and other constituents from Pilocarpus trachyllophus. Phytochemistry, v. 42, n. 3, 885-887, 1996.

ANDRADE NETO, M.; CUNHA, U.A.; MAFEZOLI, J.; SILVEIRA, E.R. Volatile constituents of Pilocarpus trachyllophus Holmes and Pilocarpus jaborandi Holmes (Rutaceae). J. Essent. Oil Res., v. 12, p. 769-774, 2000.

ANDRADE NETO, M.; CUNHA, U.A.; MAFEZOLI, J.; SILVEIRA, E.R. Volatile constituents of different populations of Pilocarpus spicatus Saint Hill. (Rutaceae) from the Northeast of Brazil. J. Essent. Oil Res., v. 14, p. 319-324, 2002.
ARTHUR, H.R.; WUI, W.H.; MA, C.N. An examination of the Rutaceae of Hong Kong (Part I), Flavonoid glycosides from Zanthoxylum species and occurence of optically active hesperidin. J. Chem. Soc., p. 632-635, 1956.

ASHWOOD-SMITH, M.J.; TOWERS, G.H.; ABRAMOWSKI, Z.; POULTON, G.A.; LIU, M. Photobiological studies with dictamnine, a furoquinoline alkaloid. Mutat. Res., v. 102, n. 4, p. 401-412, 1982.

AWAD, A.B.; ROY, R.; FINK, C.S. b-sitosterol, a plant sterol induces apoptosis and activates key caspases in MDAMB-231 human breast cancer cells. Oncol. Rep., v.10, n. 2, p. 497- 500, 2003.

BALSAM, G.; VOIGTLÄNDER, H.W. A psychotropic alkaloid from Pilocarpus organensis. Archiv. Pharm., v.311, n. 78, p. 1016-1018, 1978.

BATTERSBY, A.R.; OPENSHAW, H.T. The imidazole alkaloids. In: MANSKE, R.F.H.; HOLMES, H.L., eds. The alkaloids. San Diego: Academic Press, 1953. v. 20, p. 221-246.

BERTRAND, C.; FABRE, N.; MOULIS, C. Constituents of Pilocarpus trachylophus. Fitoterapia, v. 72, p. 844-847, 2001.

BROCHMANN-HANSSEN, E.; NUNES, M.A.; OLAH, C.K. On the biosynthesis of pilocarpine. Planta Med., v. 28, p. $1-5,1975$.

CALCAGNO, M.P.; COLL, J.; LLORIA, J.; FAINI, F.; ALONSO-AMELOT, M.E. Evaluation of synergism in feeding deterrence of some furanocoumarins on Spodoptera littoralis.J. Chem. Ecol., v. 28, n. 1, 175-191, 2002.

CHIOU, W.F.; HUANG, Y.L.; CHEN, C.F.; CHEN, C.C. Vasorelaxing effect of coumarins from Cnidium monnieri on rabbit corpus cavernosum. Planta Med., v. 67, n. 3, p. 282-284, 2001.

COMPAGNONE, R.; RODRIGUEZ, M.C. Coumarins from Pilocarpus racemosus. Fitoterapia, v. 64, n. 6, p. 557, 1993.

CORDELL, G. Introduction to alkaloids - a biogenic approach. New York: John-Willey \& Sons, 1981. $1055 \mathrm{p}$. 
CORRÊA, M.P. Dicionário de Plantas úteis do Brasil e das exóticas cultivadas. Rio de Janeiro: Imprensa Nacional, 1969. v. 4.374 p.

CORRÊA, M.P. Dicionário de Plantas úteis do Brasil e das exóticas cultivadas. Rio de Janeiro: Imprensa Nacional, 1974. v. 5.256 p.

CRAVEIRO, A.A.; ANDRADE, C.H.S.; MATOS, F.J.A.; ALENCAR, J.W. Essential oils from Brazilian Rutaceae. I Genus Pilocarpus. J. Nat. Prod., v. 42, n. 6, p. 669-671, 1979.

DEWICK, P.M. Medicinal natural products: a biosynthetic approach. New York: John-Willey \& Sons, 2000. 466 p.

EGLINTON, G.; HAMILTON, R.J.; RAPHAEL, R.A.; GONZALEZ, A.G. Hydrocarnbon constituents of the wax coatings of plants leaves: a taxonomic survey. Phytochemistry, v. 1, p. 89-102, 1962.

FIGUEIREDO, A.C.S. Achillea millefolium ssp. millefolium: produção de metebólitos secundários in vivo e in vitro. 1992. 208 p. [Tese de Doutoramento, Universidade de Lisboa].

GARG, A.; GARG, S.; ZANEVELD, L.J.D.; SINGLA, A.K. Chemistry and pharmacology of the Citrus bioflavonoid Hesperidin. Phytother. Res., v. 15, p. 655-669, 2001.

GERRARD, A.W. Alkaloid and active principle of jaborandi. Pharmaceutical Journal, v. 5, p. 865, 1875. Apud: HOLMSTED, B., WASSEN, S.H.; SCHULTES, R.E. Jaborandi: An interdisciplinary appraisal. $J$. Ethnopharmacol., v. 1, p. 3-21, 1979.

GUERREIRO, G.; FERNANDES, J.B.; VIEIRA, P.C.; DA SILVA, M.F.G.F.; RODRIGUES FILHO, E.; VICTOR, S.R.; MAGALHÃES, C.; BUENO, O.C.; HEBLING, M.J.A.; PAGNOCCA, F.C. Sesquiterpenos e cumarinas bioativos de Pilocarpus riedelianus. In: REUNIÃO ANUAL DA SOCIEDADE BRASILEIRA DE QUÍMICA, 24., Poços de Caldas, 2001. Livro de Resumos. São Paulo: SBQ, 2001. PN-008.

GOMEZ, M.A.; SÁENZ, M.T.; GARCIA, M.D.; AHUMADA, M.C.; DE LA PUERTA, R. Cytostatic activity againsty HEp-2 cells of methanolic extracts from Viscum cruciatum Sieber parasitic on Crataegus monogyna Jacq. and two isolated principles. Phytother. Res., v. 11, p. 240-242, 1997.
HE, W.; van PUYVELDE, L.; de KIMPE, N.; VERBRUGGEN, L.; ANTHONISSEN, K.; van der FLAAS, M.; BOSSLAERS, J.; MATHENGE, S.G.; MUDIDA, F.P. Chemical constituents and biological activities of Zanthoxylum usambarense. Phytother. Res., v. 16, p. 66-70, 2002.

HARDY, M.E., Sur le jaborandi (Pilocarpus pinnatus). Bulletin Societe Chimi Paris, v. 24, p. 497-500, 1875. Apud: HOLMSTED, B.; WASSEN, S.H.; SCHULTES, R.E. Jaborandi: An interdisciplinary appraisal. $J$. Ethnopharmacol., v. 1, p. 3-21, 1979.

HOLMSTED, B.; WASSEN, S.H.; SCHULTES, R.E. Jaborandi: An interdisciplinary appraisal. $J$. Ethnopharmacol., v. 1, p. 3-21, 1979.

HOROWITZ, R.M.; GENTILI, B. Flavonoids of Citrus. Tetrahedron, v. 19, p. 773-782, 1963.

HOULT, J.R.; PAYA, M. Pharmacological and biochemical actions of simple coumarins: natural products with therapeutic potential. Gen. Pharmacol., v. 27, n. 4, 713$722,1996$.

HUANG, L.; KASHIWADA, Y.; COSENTINO, L.M.; FAN, S.; CHEN, C.H.; MCPHAIL, A.T.; FUJIOKA, T.; MIHASHI, K.; LEE, K.H. Anti-Aids agents 15. Synthesis and anti-HIV activity of dihydroseselins and related analogs. J. Med. Chem., v. 37, n. 23, p. 3947- 3955, 1994.

JOWETT, H.A.D. Pilocarpine and the alkaloids of Jaborandi leaves. J. Chem. Soc., v. 77, p. 473-499, 1900.

KAASTRA, R.C. New taxa and combinations in Rutaceae. Acta Botanica Neerlandica, v. 26, n. 6, p. 471-488, 1977. Apud: SKORUPA, L.A.; SALATINO, M.L.F.; SALATINO, A. Hydrocarbons of leaf epicuticular waxes of Pilocarpus (Rutaceae): Taxonomic meaning. Biochem. Syst. Ecol., v. 26, p. 655-662, 1998.

KANES, K.; TISSERAT, B.; BERHOW, M.; VANDERCOOK, C. Phenolic composition of various tissues of Rutaceae species. Phytochemistry, v. 324, p. 967-974, 1993.

KIM, S.R.; PARK, M.J.; LEE, M.K.; SUNG, S.H.; PARK, E.J.; KIM, J.; KIM, S.Y.; OH, T.H.; MARKELONIS, G.J.; KIM, Y.C. Flavonoids of Inula britannica protect cultured cortical cells from necrotic cell death induced by glutamate. Free Radical Biol. Med., v. 32, n. 7, p. 596604, 2002. 
KOROLKOVAS, A. Dicionário Terapêutico Guanabara. Rio de Janeiro: Guanabara-Koogan, 1995/1996. p. 20.220.3.

LOUREIRO, A.A.; SILVA, M.F. Catálogo das madeiras da Amazônia. Belém: SUDAM, 1968. v. 2, p. 261-266.

MACÍAS, F.A.; GALINDO, J.C.C.; MASSANET, G.M.; RODRIGUEZ-LUIZ, F.; ZUBIA, E. Allelochemicals from Pilocarpus goudotianus leaves. J. Chem. Ecol., v. 19, n. 7, p. 1371-1379, 1993.

MAFEZOLI, J.; VIERA, P.C.; FERNANDES, J.B.; da SILVA, M.F.G.F.; DE ALBUQUERQUE, S. In vitro activity of Rutaceae species against the trypomastigote form of Trypanosoma cruzy. J. Ethnopharmacol., v. 73, p. 335-340, 2000.

MANDERFELD, M.M.; SCHAFER, H.W.; DAVIDSON, P.M.; ZOLLOTA, E.A. Isolatio and identification of antimicrobial furocoumarins from parsley. J. Food Prot., v. 60, n. 2, p. 72-77, 1997.

MARDER, M.; PALADINI, A.C. GABA A-receptor ligands of flavonoid structure. Curr. Top. Med. Chem., v. 2, p. 853-863, 2002.

MARDER, M.; VIOLA, H.; WASOWSKI, C.; FERNANDEZ, S.; MEDINA, J.H.; PALADINI, A.C. 6Methylapigenin and hesperidin: new valeriana flavonoids with activity on the CNS. Pharmacol. Biochem. Behavior, v. 75, n. 3, p. 537-545, 2003.

MAKERSBY, W.R. Oxidative stess hypothesis in Alzheimer's disease. Free Radical Biol. Med., v. 23, p. 134-147, 1997.

MEEPAGALA, K.M.; STURTZ, G.; WEDGE, D.E. Antifungal constituents of the essential oil fraction of Artemisia dracunculus L. var dracunculus. J. Agric. Food Chem., v. 50, n. 24, p. 6989-6992, 2002.

MEDINA, J.H.; VIOLA, H.; WOLFMAN, C.; MARDER, M.; WASOWKI, C.; CALVO, D. Flavonoids: a new family of benzodiazepine receptor ligands. Neurochem. Res., v. 22, n. 4, p. 419-425, 1997.

MESTER, I. The occurrence of the alkaloids in Rutaceae. Fitoterapia, v. 44, p.123-152, 1973.
MIDDLETON, E.J. The flavonoids. Trends Pharmacol. Sci., v. 8, p.335-338, 1984.

MILLER, L.J. Oral pilocarpine for radiation-induced xerostomia. Cancer Bull.(Houston), v. 45, n. 6, p. 549$550,1993$.

MOON, E.J.; LEE, Y.M.; LEE, O.H.; LEE, M.J.; LEE, S.K.; CHUNG, M.H.; PARK, Y.I.; SUNG, C.K.; CHOI, J.S.; KIM, K.W. Anovel angiogenic factor derived from Aloe vera gel: b-sitosterol, a plant sterol. Angiogenesis, v.3, n. 2, p. 117- 123, 1999.

MÜLLER, A.H.; DEGASPARI, L.R.O.; VIEIRA, P.C.; da SILVA, M.F.G.; FERNANDES, J.B.; PIRANI, J.R. 3methoxyfuranocoumarins from Pilocarpus riedelianus. Phytochemistry, v. 34, n. 2, p. 585-586, 1993.

MURAKAMI, A.; KUKI, W.; TAKAHASHI, Y.; YONEI, H.; NAKAMURA, Y.; OHTO, Y.; OHIGASHI, H.; KOSHIMIZU, K. Auraptene, a citrus coumarin, inhibits 12-O-tetradecanoylphorbol-13-acetate-induced tumor promotion in ICR mouse skin, possibly through suppression of superoxide generation in leukocytes. Jan. J. Cancer Res., v. 88, n. 5, p. 443-452, 1997.

NEGRI, G.; SALATINO, M.L.F.; SALATINO, A.; SKORUPA, L.A. An aromatic hydrocarbon from the foliar epicuticular wax of Pilocarpus jaborandi. Phytochemistry, v. 49, n. 1, p. 127-129, 1998.

NGWENDSON, J.N.; BEDIR, E.; EFANGE, S.M.; OKUNJI, C.O.; IWU, M.M.; SCHUSTER, B.G.; KHAN, I.A. Constituents of Peucedanum zenkeri seeds and their antimicrobial effects. Pharmazie, v. 58, n. 8, p. 587-589, 2003.

OGATA, M.; HOSHI, M.; URANO, S.; ENDO, T. Antioxidant activity of eugenol and related monomeric and dimeric compounds. Chem. Pharm. Bull., v. 48, n. 10, p.1467-1469, 2000.

PARK, I.K.; LEE, H.S.; LEE, S.G.; PARK, J.D.; AHN, Y.J. Inseticidal and fumigant activities of Connamomun cassia bark-derived materials against Mechoris ursulus (Coleopetra: attelabidae). J. Agric. Food Chem., v. 48, n. 6, p. 2528-2531, 2000.

PATHAK, D.; PATHAK, K.; SINGLA, A. Flavonoids as medical agents - recent advances. Fitoterapia, v. LXII, p. 317-389, 1991. 
PAVÃO F.; CASTILHO M.S.; PUPO M.T.; DIAS R.L.A.; CORREA A.G.; FERNANDES J.B.; SILVA M.F.G.F.; MAFEZOLI J.; VIEIRA P.C.; OLIVA G. Structure of Trypanosoma cruzi glycossomal glyceraldehyde-3phosphate dehydrogenase complexed with chalepin, a natural product inhibitor, at $1.95 \AA$ resolution. FEBS Lett., v. 520, p. 13-17, 2002.

PAYO HILL, A.; DOMINICIS, M.E.; OQUENDO, M.; SARDUY, R. Obtención de pilocarpina a partir de Pilocarpus racemosus Vahl. Rev. Cubana Farm., 1995. Disponível em: www.infomed.sld.cu/revistas/far/ vol29_2_95/far12295.htm.. Acesso em: 22 abr. 2001.

PINHEIRO, C.U.B. Jaborandi (Pilocarpus sp., Rutaceae): a wild species and its rapid transformation into a crop. Econ. Bot., v. 51, n. 1, p.49-58, 1997.

PIRANI, J.R. Estudos taxonômicos em Rutaceae: Revisão de Helieta e Balfourodendron (Pteleinae). Análise cladística de Pteleinae. Sinópse de Rutaceae do Brasil. São Paulo. 1999. p. irreg. [Tese de Livre docência, Instituto de Biologia, Universidade de São Paulo, São Paulo].

PYMAN, F.L. Pilosine: a new alkaloid from Pilocarpus microphyllus. J. Chem. Soc., v. 101, p. 2206-2271, 1912.

PRIYADARSINI, K.I.; GUHA, S.N.; RAO, M.N. Physicochemical properties and antioxidant activities of methoxy phenols. Free Radical Biol. Med., v. 24, n. 6, p. 933-941, 1998.

RIBA, J.; RODRIGUEZ-FORNELLS, A.; BARBANOJ, M.J. Effects of ayahuasca on sensory and sensorimotor gating in humans as measured by P50 suppression and prepulse inhibition of the startle reflex, respectively. Psychopharmacol., v. 165, n. 1, p. 18-28, 2002.

SANTOS, F.A.; CUNHA, G.M.A.; VIANE, G.S.B.; RAO, V.S.N.; MANOEL, A.N.; SILVEIRA, E.R. Antibacterial activity of essential oils from Psidium and Pilocarpus species of plants. Phytother. Res., v. 11, p. 67-69, 1997.

SANTOS, A.P.; CORNÉLIO, M.L.; LIMBERGER, R.P.; APEL, M.A.; HENRIQUES, A.T.; MORENO, P.R.H. Analysis of the Volatile Oil from Pilocarpus pennatifolius Lemmaire (Rutaceae) Leaves by GC/MS. Flavour Fragrance J., v. 19, n. 4, p. 325-326, 2004.
SAUVAIN, M.; KUNESH, N.; POISSON, J.; GANTIER, J.C.; GAYRAL, P.; DEDET, J.P. Isolation of leishmanicidal triterpenes and lignans from the Amazonian liana Dolicarpus dentatus (Dilleniaceae). Phythother. Res., v. 10, p. 1-4, 1996.

SINGH, B.; SINGH, S. Antimicrobial activity of terpenoids from Trichodesma amplexicaule Roth. Phytother. Res., v. 17, n. 7, p. 814- 816, 2003.

SHAW, C.Y.; CHEN, C.H.; HSU, C.C.; CHEN, C.C.; TSAI, Y.C. Antioxidant properties of scopoletin isolated from Sinomonium acutum. Phytother. Res., v. 17, n. 7, p. 823825, 2003.

SKORUPA, L.A. Revisão taxonômica de Pilocarpus Vahl (Rutaceae). São Paulo. 1996. 426 p. [Tese de doutoramento, Instituto de Biologia, Universidade de São Paulo].

SKORUPA, L.A.; SALATINO, M.L.F.; SALATINO, A. Hydrocarbons of leaf epicuticular waxes of Pilocarpus (Rutaceae): Taxonomic meaning. Biochem. Systemat. Ecol., v. 26, p. 655-662, 1998.

De SOUZA, R.C.; FERNANDES, J.B.; VIEIRA, P.C.; da SILVA, M.F.G.F.; RODRIGUES FILHO, E.; PIRANI, J.R. Constituintes químicos de Pilocarpus grandiflorus (Rutaceae). In: REUNIÃO ANUAL DA SOCIEDADE BRASILEIRA DE QUÍMICA, 26., Poços de Caldas, 2003. Livro de Resumos. São Paulo: SBQ, 2003, PN-203.

De SOUZA, R.C., FERNANDES, J.B., VIEIRA, P.C., Da SILVA, M.F.G.F., RODRIGUES FILHO, E., BUENO, O.C., HEBLING, M.J.A., ZANCUL, M.S.. Alcalóide furoquinolínico de Pilocarpus grandiflorus e sua ação sobre o fungo simbionte se formigas cortadeiras. In: REUNIÃO ANUAL DA SOCIEDADE BRASILEIRA DE QUÍMICA, 25., Poços de Caldas, 2002. Livro de Resumos. São Paulo: SBQ, 2002, PN-130.

De SOUZA, R.C., FERNANDES, J.B., VIEIRA, P.C., Da SILVA, M.F.G.F., RODRIGUES FILHO, E., BUENO, O.C., HEBLING, M.J.A., ZANCUL, M.S. Estudo fitoquímico de Pilocarpus grandiflorus e sua ação sobre o fungo simbionte de formigas cortadeiras. In: REUNIÃO ANUAL DA SOCIEDADE BRASILEIRA DE QUÍMICA, 24., Poços de Caldas, 2001. Livro de Resumos. São Paulo: SBQ, 2001, PN-155. 
SU, T.L.; LIN, F.W.; TENG, C.M.; CHEN, K.T.; WU, T.S. Antiplatelet aggregation principles from the stem and root bark of Melicope triphylla. Phytother. Res., v. 12, p. S74-S76, 1998.

TEDESCHI, E.; KAMIONSKY, J.; FACKLER, S.; SAREL, $\mathrm{S}$. The isolation of pure pilosine and epi-isopilosine from leaves of Pilocarpus jaborandi. Israel J. Chem., v. 11, n. 5, p. 731-733, 1973.

TENG, C.M.; LI, H.L.; YU, S.M.; WU, T.S.; HUANG, S.C.; PENG, I.S.; HUANG, T.F.A.S.I.A. $\mathrm{Ca}^{+2}$ channel blocking effects of 3 coumarin compounds, isolated from Citrus grandis, in rat thoracic aorta. Pac. J. Pharmacol., v. 7, n. 2, p. 115-120, 1992.

THIRUGNANASAMBANTHAN, P.; VISWANATHAN, S.; MYTHIRAYEE, C.; KRISHNAMURTY, V.; RAMACHANDRAN, S.; KAMESWARAN, L. Analgesic activity of certain flavone derivatives: a structure-activity study. J. Ethnopharmacol., v. 28, p. 207-214, 1990.

VALDEZ, I.H.; WOLFF, A.; ATKINSON, J.C.; MACYNSKY, A.A.; FOX, P.C. Use of pilocarpine during head and neck radiation theraphy to reduce xerostomia and salivary dysfunction. Cancer, v. 71, n. 5, p.1848-1851, 1993.
VILLASENOR, I.M.; ANGELADA, J.; CANLAS, A.P.; ECHEGOYEN, R. Bioactivity studies on b-sitosterol and its glucoside. Phytother. Res., v. 16, n. 5, p. 417-421, 2002.

VOIGTLÄNDER, H.W.; ROSENBERG, W. Über der isopilosin und pilosin. Arch. Pharm., v. 292, p. 579-585, 1959.

WEBER, U. Ueber die Wikung des Pilocarpium muriaticum. Central. Med. Wiss., v. 44, p. 409-422, 1876. Apud: HOLMSTED, B.; WASSEN, S.H.; SCHULTES, R.E. Jaborandi: An interdisciplinary appraisal. $J$. Ethnopharmacol., v. 1, p. 3-21, 1979.

YU, S.M.; KO, F.N.; SU, M.J.; WU, T.S.; WANG, M.L.; HUANG, T.F.; TENG, C.M. Vasorelaxing effect in rat thoracic aorta caused by fraxinellone and dictamine isolated from the Chinese herb Dictamnus dasycarpus Turcz: comparison with cromakalim and $\mathrm{Ca}^{2+}$ channel blockers. Naunyn Schmiedebergs Arch. Pharmakol., v. 345, n. 3, p. 349-555, 1992.

Recebido para publicação em 01 de março de 2004. 